\title{
Phase Separation of Bose-Einstein Condensates
}

\author{
E. Timmermans \\ Institute for Atomic and Molecular Physics \\ Harvard-Smithsonian Center for Astrophysics \\ 60 Garden Street \\ Cambridge, MA 02138
}

(March 20, 2018)

\begin{abstract}
The zero-temperature system of two dilute overlapping Bose-Einstein condensates is unstable against long wavelength excitations if the interaction strength between the distinguishable bosons exceeds the geometric mean of the like-boson interaction strengths. If the condensates attract each other, the instability is similar to the instability of the negative scattering length condensates. If the condensates repel, they separate spatially into condensates of equal pressure. We estimate the boundary size, surface tension and energy of the phase separated condensate system and we discuss the implications for double condensates in atomic traps.
\end{abstract}

PACS numbers(s):03.75.Fi, 05.30.Jp, 32.80Pj, 67.90.+z 
As dilute gases, the atomic trap Bose-Einstein condensates [1] occupy a unique position among the superfluid systems. One intriguing consequence of their dilute gas nature is the prospect of studying condensate mixtures, which are the first experimentally realizable bosonic superfluid mixtures [2]- [6] (the only other mixture of superfluids is the fermionboson ${ }^{3} \mathrm{He}^{-4} \mathrm{He}$ system). Understandably, this prospect has attracted interest [7]- [10], and recently the first observation of overlapping condensates was reported [8].

In this paper, we study if and when zero temperature dilute condensates overlap. We find that the homogeneous overlapping condensate system is unstable against long wavelength excitations if the strength of the interaction between the distinguishable bosons exceeds the geometric mean of the like-boson interaction strengths. In that case, two repelling condensates spatially separate into single condensates of equal pressure. The condensates still partially overlap in the boundary region that separates them. We estimate the size of the boundary region, as well as the corresponding surface tension. We show how the homogenous treatment may be generalized to describe phase-separated large double condensate systems in a trap.

The instability of the overlapping condensates manifests itself in the energy dispersion of the elementary excitations [10], as we show below. The wavefunctions, $\phi_{1}$ and $\phi_{2}$, of two interacting condensates satisfy coupled Gross-Pitaevski equations:

$$
\begin{aligned}
& i \hbar \dot{\phi}_{1}=\left[-\frac{\hbar^{2} \nabla^{2}}{2 m_{1}}-\mu_{1}+\lambda_{1}\left|\phi_{1}\right|^{2}\right] \phi_{1}+\lambda\left|\phi_{2}\right|^{2} \phi_{1} \\
& i \hbar \dot{\phi}_{2}=\left[-\frac{\hbar^{2} \nabla^{2}}{2 m_{2}}-\mu_{2}+\lambda_{2}\left|\phi_{2}\right|^{2}\right] \phi_{2}+\lambda\left|\phi_{1}\right|^{2} \phi_{2},
\end{aligned}
$$

where $\mu_{j}(j=1,2)$ represents the chemical potential of the $j$-bosons. The interaction strength values, $\lambda_{j}$ and $\lambda$, are determined by the scattering lengths for binary collisions of distinguishable bosons: $\lambda_{j}=4 \pi \hbar^{2} a_{j} / m_{j}$ and $\lambda=2 \pi \hbar^{2} a / m_{\text {red }}$, where $m_{\text {red }}^{-1}=m_{1}^{-1}+m_{2}^{-1}$. The excitations of the static homogeneous condensates, $\phi_{j}(\mathbf{r}, t)=\phi_{j}^{(0)}$, are described by fluctuations of the fields, $\phi_{j}(\mathbf{r}, t)=\phi_{j}^{(0)}+\delta \phi_{j}$, which evolve according to the Gross-Pitaevski equations (11), linearized in $\delta \phi$ and $\delta \phi^{*}$ [11]. Decomposing the field fluctuations into Fourier components, $\delta \phi_{j}=\sum_{\mathbf{k}} c_{j, \mathbf{k}} \exp (i \mathbf{k} \cdot \mathbf{r})$, we obtain the equations of motion for the c-amplitudes, 


$$
\begin{array}{r}
i \hbar \dot{c}_{1, \mathbf{k}}=\left[k^{2} / 2 m_{1}+\lambda_{1} n_{1}\right] c_{1, \mathbf{k}}+\lambda_{1} \phi_{1}^{(0)} c_{1,-\mathbf{k}}^{*} \\
+\lambda \phi_{1}^{(0)}\left[\phi_{2}^{(0) *} c_{2, \mathbf{k}}+\phi_{2}^{(0)} c_{2,-\mathbf{k}}^{*}\right]
\end{array}
$$

where $n_{j}=\left|\phi_{j}^{(0)}\right|^{2}$ and where we have used that $\dot{\phi}_{j}^{0}=0$. A second equation for $i \hbar \dot{c}_{2}$ is obtained by exchanging the 1 and 2 subscripts. Alternatively, we can introduce the phase and density of the condensate field, $\phi=\sqrt{\rho} \exp (i \theta)$, the fluctuations of which, $\rho=n+\delta \rho$ and $\theta=\theta^{(0)}+\delta \theta$, account for the field fluctuations, $\delta \phi=\phi^{(0)}[\delta \rho / 2 n+i \delta \theta]$. The last term of Eq.(22) then represents a density fluctuation, $\delta \rho_{\mathbf{k}}=\left[\phi^{(0) *} c_{\mathbf{k}}+\phi^{(0)} c_{-\mathbf{k}}^{*}\right]$ whereas the difference, $\delta \Pi_{\mathbf{k}}=\left[\phi^{(0) *} c_{\mathbf{k}}-\phi^{(0)} c_{-\mathbf{k}}^{*}\right] / 2 i$, represents a phase fluctuation. Multiplying Eq. (2) by $\phi_{1}^{(0) *}$ and adding and subtracting the resulting equalities with the complex conjugate equation, the $\delta \Pi$ and $\delta \rho$-equations of motion follow:

$$
\begin{aligned}
\hbar \delta \dot{\rho}_{1, \mathbf{k}} & =2\left[\hbar^{2} k^{2} / 2 m_{1}\right] \delta \Pi_{1, \mathbf{k}}, \\
\hbar \delta \dot{\Pi}_{1, \mathbf{k}} & =-\frac{1}{2}\left[\hbar^{2} k^{2} / 2 m_{1}+2 \lambda_{1} n_{1}\right] \delta \rho_{1, \mathbf{k}}-\lambda n_{1} \delta \rho_{2, \mathbf{k}} .
\end{aligned}
$$

Thus, the phase fluctuations of one condensate couple to the density fluctuations of the other. We cancel out the dependence on the phase fluctuations by taking the derivative of the first equation in Eqs. (3) with respect to time and by substituting $\delta \dot{\Pi}_{\mathbf{k}}$ from the second equation. With $\delta \rho_{\mathbf{k}}(t)=\delta \rho_{\mathbf{k}} \cos \left(\Omega_{\mathbf{k}} t\right)$, we find the normal mode equations for the coupled density fluctuations,

$$
\begin{aligned}
-\Omega_{\mathbf{k}}^{2} \delta \rho_{1, \mathbf{k}} & =-\omega_{1, \mathbf{k}}^{2} \delta \rho_{1, \mathbf{k}}-\lambda n_{1} \frac{k^{2}}{m_{1}} \delta \rho_{2, \mathbf{k}} \quad, \\
-\Omega_{\mathbf{k}}^{2} \delta \rho_{2, \mathbf{k}} & =-\omega_{2, \mathbf{k}}^{2} \delta \rho_{2, \mathbf{k}}-\lambda n_{2} \frac{k^{2}}{m_{2}} \delta \rho_{1, \mathbf{k}},
\end{aligned}
$$

where $\hbar \omega_{j, \mathbf{k}}=\sqrt{\left(\hbar^{2} k^{2} / 2 m_{j}\right)^{2}+\left(\hbar^{2} k^{2} / m_{j}\right) n_{j} \lambda_{j}}$ (we assume $\left.\lambda_{j}>0\right)$ denotes the usual single condensate Bogoliubov dispersion. Requiring Eq.(任) to have non-trivial solutions gives the dispersions of the double-condensate excitations:

$$
\begin{aligned}
\Omega_{ \pm, \mathbf{k}}^{2}= & \frac{\left[\omega_{1, \mathbf{k}}^{2}+\omega_{2, \mathbf{k}}^{2}\right]}{2} \\
& \pm \frac{\sqrt{\left[\omega_{1, \mathbf{k}}^{2}-\omega_{2, \mathbf{k}}^{2}\right]^{2}+4\left(\lambda^{2} / \lambda_{1} \lambda_{2}\right) c_{1}^{2} c_{2}^{2} k^{4}}}{2} .
\end{aligned}
$$


where $c_{j}$ is the sound velocity of the $j$-condensate, $c_{j}=\sqrt{n_{j} \lambda_{j} / m_{j}}$. The \pm -sign in the subscript, $\Omega_{ \pm}$, corresponds to the choice of + or - in Eq.(5). The implications for the physics of the overlapping condensate systems are profound: the near-equilibrium dynamics and thermodynamics of the two-condensate system are essentially the dynamics and thermodynamics of two sets of nearly non-interacting quasi-particles with energies $\Omega_{ \pm \mathbf{k}}$.

We are concerned with the stability of the overlapping condensate system to which purpose we consider the long wavelength limit, $k \rightarrow 0$, of the $\Omega$-dispersion relations. With the long wavelength Bogoliubov energies, $\omega_{j, \mathbf{q}} \approx c_{j} q$, we find that the double condensate dispersions of Eq.(5) are also phonon-like, $\Omega_{ \pm, \mathbf{k}} \approx c_{ \pm} k(k \rightarrow 0)$, with 'sound velocities' $c_{+}$ and $c_{-}$determined by

$$
c_{ \pm}^{2}=\frac{\left[c_{1}^{2}+c_{2}^{2}\right] \pm \sqrt{\left[c_{1}^{2}-c_{2}^{2}\right]^{2}+4\left(\lambda^{2} / \lambda_{1} \lambda_{2}\right) c_{1}^{2} c_{2}^{2}}}{2}
$$

If $\lambda^{2}>\lambda_{1} \lambda_{2}$, the inter-condensate interaction repels the energy levels so strongly that $c_{-}^{2}$ becomes negative. Studying the normal mode frequencies of small deviations is a standard test of stability and $c_{-}^{2}<0$ indicates that the overlapping condensate system is unstable against long wavelength excitations.

The thermodynamic properties of the double condensate are determined by minimizing the free energy $F$. If the spatial variations of the condensates are slow and the kinetic energy contributions may be neglected, the zero-temperature free energy is the integral over the free energy density $F(\mathbf{r})$,

$$
\begin{array}{r}
F(\mathbf{r})=\frac{\lambda_{1}}{2} n_{1}^{2}(\mathbf{r})+\frac{\lambda_{2}}{2} n_{2}^{2}(\mathbf{r})+\lambda n_{1}(\mathbf{r}) n_{2}(\mathbf{r}) \\
-\mu_{1}(\mathbf{r}) n_{1}(\mathbf{r})-\mu_{2}(\mathbf{r}) n_{2}(\mathbf{r})
\end{array}
$$

where the effective chemical potentials $\mu_{j}(\mathbf{r})$ include the external potentials $v_{j}(\mathbf{r})$ experienced by the $j$ - bosons, $\mu_{j}(\mathbf{r})=\mu_{j}-v_{j}(\mathbf{r})$. Minimizing $\mathrm{F}$ with respect to the densities, $\delta F / \delta n_{j}(\mathbf{r})=$ 0 , gives the Thomas-Fermi equations,

$$
\begin{aligned}
& \mu_{1}(\mathbf{r})=\lambda_{1} n_{1}(\mathbf{r})+\lambda n_{2}(\mathbf{r}) \\
& \mu_{2}(\mathbf{r})=\lambda_{2} n_{2}(\mathbf{r})+\lambda n_{1}(\mathbf{r})
\end{aligned}
$$


When $v_{j}(\mathbf{r})=0$, the Thomas-Fermi condensate densities of Eq.(8) are homogeneous. However, equating first-order derivatives to zero, only gives a minimum provided the second-order derivatives satisfy $\left(\partial^{2} F / \partial n_{j}^{2}\right)>0$ and $\left(\partial^{2} F / \partial n_{1}^{2}\right)\left(\partial^{2} F / \partial n_{2}^{2}\right)-\left(\partial^{2} F / \partial n_{1} \partial n_{2}\right)^{2}>0$. The latter condition implies that the Thomas-Fermi equations (8) only gives a minimum provided the stability criterion, $\lambda^{2}<\lambda_{1} \lambda_{2}$, is satisfied.

To see that 'strongly' repulsive condensates, $\lambda>\sqrt{\lambda_{1} \lambda_{2}}$, lower their free energy by distributing the condensates inhomogeneously, we write the free energy density of Eq.(7) in the absence of external potentials, $v_{j}(\mathbf{r})=0$, as

$$
\begin{aligned}
F(\mathbf{r})=\frac{\lambda_{1}}{2} & {\left[n_{1}(\mathbf{r})+n_{2}(\mathbf{r}) \sqrt{\lambda_{2} / \lambda_{1}}\right]^{2} } \\
+ & {\left[\lambda-\sqrt{\lambda_{1} \lambda_{2}}\right] n_{1}(\mathbf{r}) n_{2}(\mathbf{r}) } \\
& -\mu_{1} n_{1}(\mathbf{r})-\mu_{2} n_{2}(\mathbf{r}) .
\end{aligned}
$$

Starting from the homogeneous overlapping condensate system, redistributing bosons 1 and 2 spatially while keeping $\left[n_{1}(\mathbf{r})+n_{2}(\mathbf{r}) \sqrt{\lambda_{2} / \lambda_{1}}\right]$ constant over space, can lower the energy by decreasing the overlap integral $\int d^{3} r n_{1}(\mathbf{r}) n_{2}(\mathbf{r})$. The lowest value is reached by spatially separating the two condensates. In that case, although the hamiltonian is translationally invariant $\left(v_{j}(\mathbf{r})=0\right)$, the double condensate is not: the double condensate system spontaneously breaks translational symmetry. Note that the phase separation is consistent with the above discussed dynamical instability - the separated condensate system has no region that can be described locally as two homogeneous condensates. In contrast, strongly attractive condensates $\left(\lambda<0, \lambda^{2}>\lambda_{1} \lambda_{2}\right)$ decrease the free energy by increasing their mutual overlap and overlapping condensates are unstable. This is similar to the behavior of single condensates of negative scattering length, and we expect the strongly attractively double condensates to be similarly unstable. The same analogy suggests that a confining potential might give stable or metastable strongly attractive double condensates.

The free energy of the phase separated condensate system in a macroscopic volume $V$, is the sum of the single condensate free energies, condensate 1 confined to a volume $V_{1}$, and condensate 2 confined to $V-V_{1}$. Minimizing the total free energy with respect to $V_{1}$ gives 
the equilibrium condition of equal pressures exerted by both condensates. With the pressure $P_{j}=\lambda_{j} n_{j, s}^{2} / 2$, where $n_{j, s}$ denotes the density within the separated condensates, we are lead to the equivalent condition for the condensate densities $n_{1, s}=n_{2, s} \sqrt{\lambda_{2} / \lambda_{1}}$.

We note that the assumption of slowly varying condensate wavefunctions, necessary to justify neglecting the kinetic energy in the free energy of Eq.(77), is violated at the boundary of the two condensates. In fact, an infinitely sharp boundary gives an infinite kinetic energy contribution. The effect of the kinetic energy is then to give a boundary region of finite size $\mathrm{b}$, in which the wavefunctions smoothly tend to zero as the condensates cross the boundary. We assume that the condensates are so large that the boundary region which separates them can be approximated locally as a planar region with densities that vary spatially as functions of the coordinate $z$ with the $z$-axis perpendicular to the boundary surface of area $A$. The kinetic energy contribution, $E_{k i n}(b)$, is then approximately equal to $E_{k i n}(b) \approx\left(A \hbar^{2} n_{1, s} / 2 m_{1} b\right)\left[1+\sqrt{\lambda_{1} / \lambda_{2}}\left(m_{1} / m_{2}\right)\right]$, where we used that $\left(n_{2, s} / n_{1, s}\right)=\sqrt{\lambda_{1} / \lambda_{2}}$. The overlap of the condensates in the boundary region increases the interaction energy by an amount $E_{\text {int }}(b)$, which we estimate by modeling the condensate densities in the boundary region, $z \in(0, b)$, crudely as $n_{1}(z) \approx n_{1, s}(b-z) / b$ and $n_{2}(z) \approx n_{2, s} z / b$. With Eq.(9), we find that $E_{\text {int }}(b) \approx A\left(\lambda-\sqrt{\lambda_{1} \lambda_{2}}\right) n_{1, s} n_{2, s} b / 6$. To estimate the actual boundary size $\bar{b}$, we minimize the boundary energy, $E_{b}(b)=E_{k i n}(b)+E_{\text {int }}(b)$ with respect to $b$ and find

$$
\bar{b}=2 l_{1} \sqrt{3} \sqrt{\frac{\left[1+\left(m_{1} / m_{2}\right) \sqrt{\lambda_{1} / \lambda_{2}}\right]}{\left[\lambda / \sqrt{\lambda_{1} \lambda_{2}}-1\right]}},
$$

where $l_{1}$ is the coherence length of condensate $1, l_{1}=\hbar / \sqrt{4 m_{1} n_{1, s} \lambda_{1}}$. The boundary contribution to the energy is a surface energy $E_{b}(\bar{b})=\sigma A$, where the surface tension $\sigma$ is proportional to the coherence length $l_{1}$, to the pressure $P_{1}, P_{1}=n_{1, s}^{2} \lambda_{1} / 2$, and to a dimensionless constant $\Sigma_{1}$ which depends solely on the mass and interaction strength ratios, $\Sigma_{1}=4 \sqrt{\left[1+\left(m_{1} / m_{2}\right) \sqrt{\lambda_{1} / \lambda_{2}}\right]\left[\lambda / \sqrt{\lambda_{1} \lambda_{2}}-1\right]} / \sqrt{3}, \sigma=l_{1} P_{1} \Sigma_{1}$.

In the absence of external potentials, a 'droplet' of condensate 1 immersed in a much larger condensate 2 of density $n_{2}$ minimizes $E_{b}$ by taking on the shape of a sphere of radius $R, V_{1}=(4 \pi / 3) R^{3}$. We can now imagine creating the double condensate system starting 
from a single condensate of type 2 and replacing condensate 2 bosons in the droplet volume $V_{1}$ by condensate 1 bosons. The energy $\Delta E$ required in the replacement is equal to $\Delta E=$ $\left[\lambda_{1, s} n_{1}^{2} / 2-\lambda_{2} n_{2, s}^{2} / 2\right] V_{1}+E_{b}$. Minimizing the 'replacement energy' $\Delta E$ with respect to $V_{1}$ and realizing that $E_{b} \propto V_{1}^{2 / 3}$, we find

$$
\frac{\lambda_{1} n_{1, s}^{2}}{2}=\frac{\lambda_{2} n_{2, s}^{2}}{2}+\frac{2}{3} \frac{E_{b}}{V_{1}} .
$$

The previous result, $\lambda_{1} n_{1, s}^{2} / 2=\lambda_{2} n_{2, s}^{2} / 2$, obtained by ignoring the boundary energy, is accurate provided the size of the droplet exceeds $R_{s}=2 \sigma / P_{1}=2 l_{1} \Sigma_{1}$. The energy per droplet particle, $\Delta E / N_{1}$, with Eq.(11) is equal to $\Delta E / N_{1}=5 E_{b} / V_{1}=5 \sigma /\left[R n_{1, s}\right]$, a function that decreases monotonically as $N_{1}$ increases. Consequently, splitting up the droplet into smaller droplets further increases the free energy and it is energetically favorable for condensate 1 to gather in a single region of space (i.e. real space condensation).

To describe separated double condensates in traps, we subtract the overlap term, $\lambda n_{1} n_{2}$, and include the boundary surface energy in the expression of the free energy (Eq.(7)). The validity of this description rests on two conditions: 1. the local coherence length within each condensate is much less than the length scale on which the condensates vary spatially and 2 . the change of the potential energy across the inter-condensate boundary, $\left|\mathbf{f}_{j}\right| \bar{b}$, where $\mathbf{f}_{j}=-\nabla v_{j}$ represents the external force experienced by bosons $\mathrm{j}$ near the boundary, is much less than the local chemical potential $\left|\mathbf{f}_{j}\right| \bar{b}<<\lambda_{j} n_{j, s}(j=1,2)$. If these conditions are satisfied, the physics of the phase separation is similar to the above $v_{j}(\mathbf{r})=0$-case, and we can answer interesting questions regarding trapped phase separated condensates. For instance: if we add a droplet of condensate 1 to a trapped condensate 2, does it 'sink' to the middle of the trap, or does it remain 'floating' on the surface of condensate 2? For the sake of simplicity, we assume that the size of the droplet is large enough to neglect the boundary surface energy and small enough to neglect the spatial variation of the density inside the droplet. Then, the previously defined 'replacement energy' $\Delta E$ depends on the center of mass position $\mathbf{R}$ of the droplet through the external potentials, $v_{1}(\mathbf{r})=v(\mathbf{r})$, and $v_{2}(\mathbf{r})=\alpha v(\mathbf{r})$. Since the pressures inside and outside the droplet are equal, $\lambda_{1} n_{1,2}^{2} / 2-\lambda_{2} n_{2, s}^{2} / 2 \approx 0$, we find that 


$$
\begin{aligned}
\Delta E(\mathbf{R}) & =\int_{V_{1}}\left[n_{1}(\mathbf{r}) v_{1}(\mathbf{r})-n_{2}(\mathbf{r}) v_{2}(\mathbf{r})\right] d^{3} r \\
& \approx N_{1} v(\mathbf{R})\left[1-\alpha \sqrt{\lambda_{1} / \lambda_{2}}\right] .
\end{aligned}
$$

Thus, even though both bosons experience a trapping potential, if $\alpha \sqrt{\lambda_{1} / \lambda_{2}}>1$, the force on the droplet, $-N_{1}\left[1-\alpha \sqrt{\lambda_{1} / \lambda_{2}}\right] \nabla v$, is directed outwards : the droplet 'floats'.

The thinner the layer of the floating condensate, the more important are the effects of the boundary surface energy. Indeed, with a few condensate 1 particles, the energy will be minimized by covering only part of the surface of condensate 2 (thereby reducing the surface boundary energy). Here, we only consider the case where enough bosons have been added for condensate 1 to 'wrap' around condenate 2, and we can ignore boundary surface energy effects. Within the single condensate regions, the condensates are described in the Thomas-Fermi approximation of Eq.(8) (putting $\lambda=0$ ), $n_{1}(\mathbf{r})=\left[\mu_{1}-v(\mathbf{r})\right] / \lambda_{1}$ and $n_{2}(\mathbf{r})=\alpha\left[\mu_{2}-v(\mathbf{r})\right] / \lambda_{2}$, where $\mu_{1}$ and $\alpha \mu_{2}$ are the chemical potentials. In that case, the boundary surface is the equipotential surface $v(\mathbf{R})=\mu_{b}$ where the pressures of both condensates, $\lambda_{j} n_{j}^{2}(\mathbf{r}) / 2$, are equal. This leads to

$$
\mu_{b}=\mu_{2}-\frac{\left[\mu_{1}-\mu_{2}\right]}{\left[\alpha \sqrt{\lambda_{1} / \lambda_{2}}-1\right]} .
$$

In figure 1, we show a typical density profile for two separated condensates in a spherically symmetric trap. With $\lambda_{j} n_{j}\left(R_{b}\right)=\mu_{j}-v_{j}\left(R_{b}\right)$, we find for the case shown in Fig.(1) that $\mu_{1}-\mu_{2}=\lambda_{1} n_{1}\left(R_{b}\right)-\lambda_{2} n_{2}\left(R_{b}\right) / \alpha>0$. Using $\left[n_{1}\left(R_{b}\right) / n_{2}\left(R_{b}\right)\right]=\sqrt{\lambda_{2} / \lambda_{1}}$ we then find that $\alpha \sqrt{\lambda_{1} / \lambda_{2}}>1$, so that condensate 1 should indeed float on top of condensate 2. Of course, the experimentally relevant quantities are the number of boson particles, $N_{1}$ and $N_{2}$, rather than $\mu_{1}$ and $\mu_{2}$. The chemical potentials can be determined by inverting $N_{1}=\int_{V_{1}} d^{3} r n_{1}(\mathbf{r})$ and $N_{2}=\int_{V_{2}} d^{3} r n_{2}(\mathbf{r})$, where the boundary between $V_{1}$ and $V_{2}$ is defined by Eq.(13), to give $N_{j}\left(\mu_{1}, \mu_{2}\right)$.

In summary, we have shown that the homogeneous overlapping double condensate system with strong inter-condensate interactions $\left(\lambda^{2}>\lambda_{1} \lambda_{2}\right)$ is unstable. In that case, attractive condensates $(\lambda<0)$ collapse and repelling condensates $(\lambda>0)$ separate spatially. In 
between the separated condensates is a region of partial overlap. We have estimated the size of this region, as well as the resulting surface tension. Finally, we briefly discussed phase separation of large condensates in atomic traps.

The phase separation suggests many experimental applications. For instance, one can use a condensate to spatially confine a droplet of a different condensate in the middle of a trap. Using light that is resonant with the droplet atoms, the droplet can be displaced and its subsequent motion inside the confining condensate can be observed. If the motion is undamped, we have a direct observation of superfluidity. To further motivate such experiments, we mention that damping generally could occur at velocities less than the sound velocity of the confining condensate because of the creation of superfluid vortices etc... Furthermore, it is interesting to note that schemes have been proposed to continuously alter the interaction strengths, either by using light [13], or by varying the bias field of the magnetic traps [14], so that it might be possible in the future to observe phase separation in real time as the interaction strengths are altered.

The author gratefully acknowledges fruitful interactions with Dr. P. Tommasini, Prof. E. Heller, Prof. A. Dalgarno and Prof. K. Huang. The work of the author is supported by the NSF through a grant for the Institute for Atomic and Molecular Physics at Harvard University and Smithsonian Astrophysical Observatory. 


\section{REFERENCES}

[1] K. B. Davis et al., Phys. Rev. Lett. 75,3969 (1995); M. H. Anderson et al., Science, 269, 198 (1995); C. C. Bradley et al., Phys. Rev. Lett. 75, 1687 (1995).

[2] Previously, the only candidate for creating interpenetrating bosonic superfluids seemed to be a mixture of ${ }^{4} \mathrm{He}$ and ${ }^{6} \mathrm{He}$. Unfortunately, the short lifetime of the ${ }^{6} \mathrm{He}$-isotope $(\sim 0.8$ seconds $)$ did not allow cooling to sufficiently low temperatures. Nevertheless, the prospect motivated theoretical work that is relevant to our paper [3], [4], [5] and [6].

[3] I. M. Khalatnikov, Zh. Eksp. Teor. Fiz. 32, 653 (1957) [ Sov. Phys. - JETP 5, 542 (1957)].

[4] W. H. Bassichis, Phys. Rev. A 134, 543 (1964).

[5] Y. A. Nepomnnyashchii, Zh. Eksp. Teor. Fiz. 70, 1070 (1976) [Sov. Phys. - JETP 43, 559 (1976)]; Teor. Mat. Fiz. 20, 399 (1974).

[6] W. B. Colson and Alexander L. Fetter, J. Low Temp. Phys. 33, 231 (1978).

[7] The overlapping double atomic condensate systems were first discussed by T.-L. Ho and V.B. Shenoy, Phys. Rev. Lett. 77, 3276 (1996).

[8] C. J. Myatt et al., Phys. Rev. Lett. 78, 586 (1997).

[9] B. D. Esry et al., Phys. Rev. Lett. 78, 3594 (1997).

[10] The dispersion for overlapping condensate systems was first obtained for boson particles of the same mass by R. Graham and D. Walls, cond-mat/9611111.

[11] The same equations can be obtained from the Heisenberg equations of motion, neglecting the interactions between excited particles.

[12] E. Timmermans, P. Tommasini and K. Huang, Phys. Rev. A, 55, 3645 (1997).

[13] P. O. Fedichev, Yu. Kagan, G. V. Shlyapnikov, and J. T. M. Walraven, Phys. Rev. Lett. 
77, 2913 (1996).

[14] E. Tiesinga, B. J. Verhaar, and H. T. Stoof, Phys. Rev. A, 47, 4114 (1993). 


\section{Figure Captions}

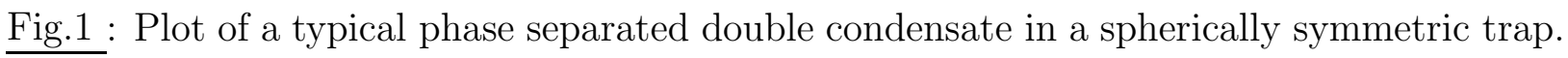
In reality, the boundary of condensates 1 and 2 (at $R=R_{b}$ where $v\left(R_{b}\right)=\mu_{b}$ ) is not infinitely sharp and the condensates overlap over a region of size $b$ that is estimated in the text. 